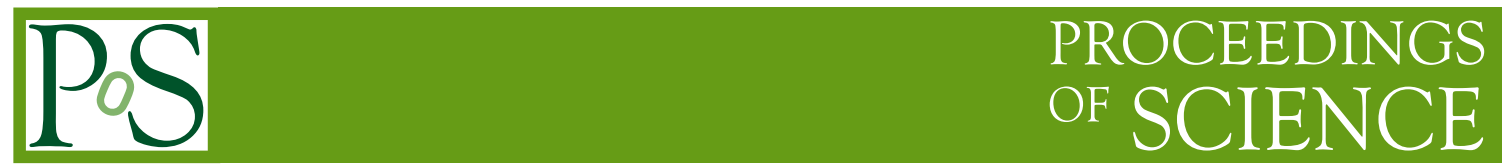

\title{
Preface QCD Evolution Workshop 2016
}

\section{Leonard Gamberg}

Penn State University Berks

E-mail: lpg10epsu. edu

\section{Alexei Prokudin}

Penn State University Berks \& Jefferson Lab

E-mail: prokudinajlab.org

\section{Anatoly Radyushkin}

Old Dominian University \& Jefferson Lab

E-mail: radyushejlab.org

These are the Proceedings of the QCD Evolution Workshop 2016, which was jointly organized by Vrije Universiteit Amsterdam, Groningen University and the National Institute for Subatomic Physics (Nikhef). This $6^{\text {th }}$ annual workshop took place at Nikhef in Amsterdam from May 30 through June 03, 2016.

QCD Evolution 2016

May 30-June 03, 2016

National Institute for Subatomic Physics (Nikhef) Amsterdam, The Netherlands 


\section{Preface}

The QCD Evolution 2016 workshop was held at the National Institute for Subatomic Physics (Nikhef) in Amsterdam, May 30 through June 3, 2016. The workshop was organized jointly by the Vrije Universiteit Amsterdam, Groningen University and the National Institute for Subatomic Physics (Nikhef) with additional support from the European Research Council (ERC), The Foundation for Fundamental Research on Matter (FOM), Los Alamos National Lab, and Pennsylvania State University-Berks.

The 2016 workshop is a continuation of the series held during five consecutive years, 2011, 2012, 2013, 2015 at Jefferson Lab, and 2014 in Santa Fe, New Mexico which organized by members of the T-2 Division at Los Alamos National Lab.

The rapid developments in our understanding of the evolution of parton distributions (PDFs) including low- $x$, transverse momentum parton distribution and fragmentation functions (TMDs), generalized parton distribution functions (GPDs), higher-twist correlation functions, and the associated progress in perturbative QCD, lattice QCD, and soft collinear effective field theory (SCET), were the focus of this year's meeting. Special attention was also given to participation by young researchers, postdocs, students. Also, many of the topics covered are of special importance for a future Electron Ion Collider (EIC).

This year's workshop attracted 65 registered participants. The Organizing Committee thanks the speakers and all those who participated in the conference for the stimulating atmosphere that prevailed. We trust the written versions of the talks presented in the Proceedings of Science (PoS) will capture the exciting physics topics covered at the workshop. In this preface you will find an image of the workshop poster and photograph of the conference participants.

Thanks are due to the people who worked hard to organize the conference and make it a success; in particular the local organizers of QCD Evolution Workshop 2016, Daniel Boer (cochair), Piet Mulders (co-chair), Alexei Prokudin (co-chair), Andrea Signori, and Tomas Kasemets. We express our special thanks to, Marja Herronen for all her organizational efforts before and during the workshop. We also express our sincere appreciation to Dr. Keith Hillkirk, Chancellor of Penn State University Berks, for support from the Penn State University-Berks Undesignated Gift Fund.

Leonard Gamberg Alexei Prokudin

Anatoly Radyushkin

30 December 2016 


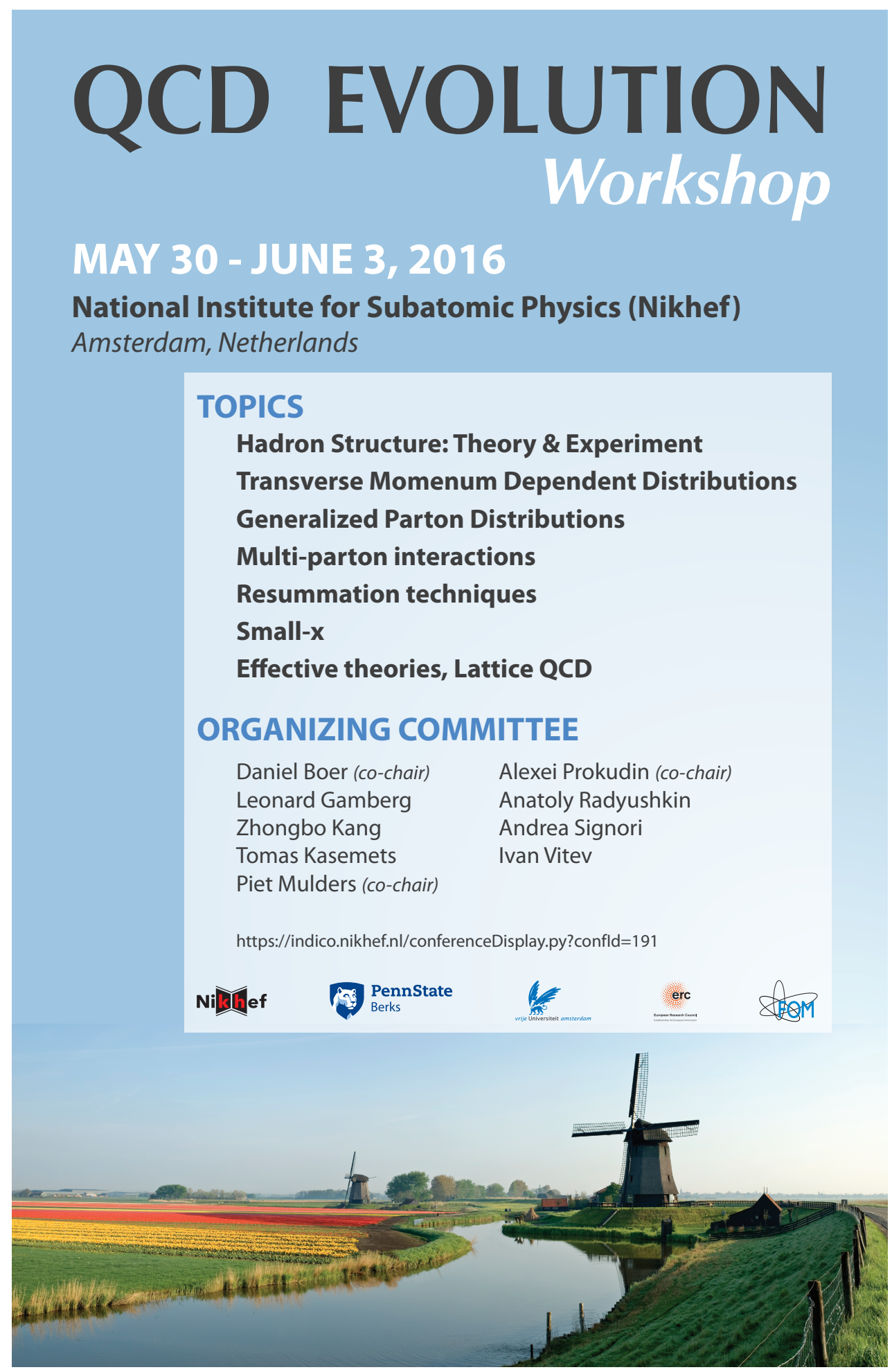




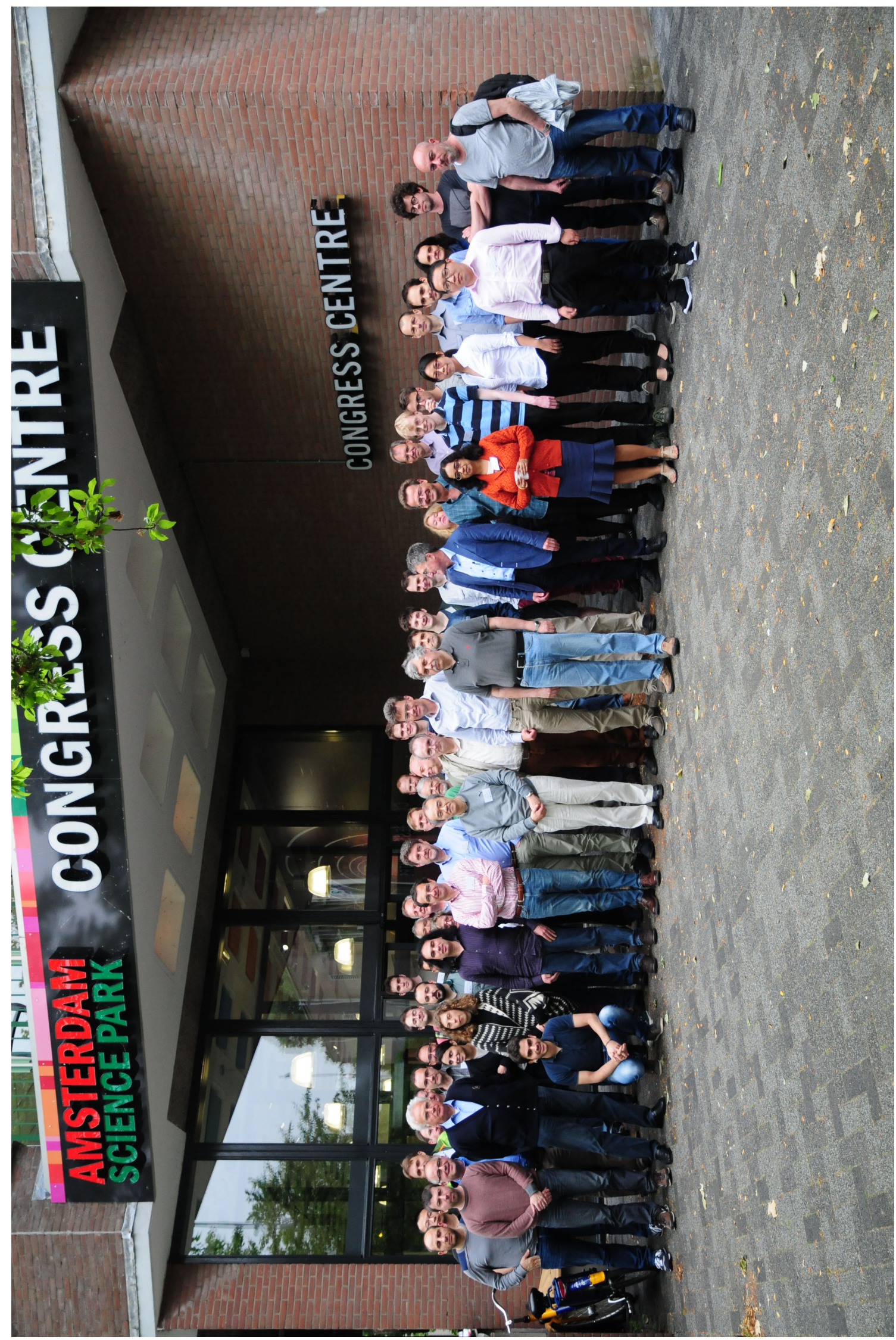

Figure 1: QCD Evolution 2016 Participants 